\title{
CONTRIBUiçÃo AO CONHECIMENTO TAXONÔMICO DE Terebellidae e Trichobranchidae (Annelida: Polychaeta) DA REgIÃo SUDESTE-SUL dO BRASIL
}

Dissertação apresentada ao Instituto de Biociências da Universidade de São Paulo, para obtenção do Título de Mestre em Ciências, na Área de Zoologia.

Orientador: Prof. Dr. João Miguel de Matos Nogueira

São Paulo

2008 


\section{RESUMO}

A presente Dissertação é resultado de um levantamento taxonômico das espécies de poliquetas das famílias Terebellidae e Trichobranchidae, coletadas pelos projetos "A Biodiversidade de Poliquetas (Annelida: Polychaeta) em Costões Rochosos ao Longo do Estado de São Paulo", "Temático BIOTA/FAPESP/Biodiversidade Bêntica Marinha no Estado de São Paulo (=Temático BIOTA/FAPESP/Bentos Marinho)" e "REVIZEE/Score Sul/Bentos", abrangendo desde a zona entremarés a ambientes de profundidade, a fim de enriquecer 0 conhecimento taxonômico das famílias Terebellidae e Trichobranchidae no litoral Sudeste e Sul do Brasil.

As identificações foram feitas segundo caracteres morfológicos, os exemplares foram observados aos microscópios óptico, estereoscópico e, em alguns casos, eletrônico de varredura, com o auxílio de bibliografia especializada. Chaves de identificação e descrições detalhadas, com pranchas ilustrativas diagnósticas, foram elaboradas para cada espécie.

No total, foram identificados 8386 indivíduos pertencentes a 15 gêneros e 21 espécies, sendo 14 gêneros e 18 espécies da família Terebellidae, subfamílias Terebellinae, Thelepodinae e Polycirrinae, e 1 gênero e 3 espécies da família Trichobranchidae. Foram encontradas 6 espécies de terebelídeos novas para a ciência: Amaeana sp., Phisidia rubra Nogueira \& Alves, 2006, Polycirrus sp., Pseudostreblosoma brevitentaculatum Nogueira \& Alves, 2006, Terebella sp. 1 e Terebella sp. 2. O gênero Phisidia Saint-Joseph, 1894 é nova ocorrência para a costa do Brasil.

Assim, a presente Dissertação pode, também, ser usada como um manual de identificação que auxiliará pesquisadores, inclusive não-especialistas, na identificação de parte das espécies de Terebellidae e Trichobranchidae das regiões Sudeste-Sul do Brasil. Espera-se também que auxilie pesquisadores interessados em identificar espécies de locais não abrangidos pelos três projetos aqui analisados, assim como em estudos ecológicos e biogeográficos, ampliando, assim, o conhecimento da biodiversidade dos Terebellidae e Trichobranchidae presentes no Brasil e no mundo. 


\section{ABSTRACT}

The present Dissertation is the result of a taxonomic survey on the species of polychaetes belonging to the families Terebellidae and Trichobranchidae collected by the projects "Biodiversity of Polychaetes on Rocky Shores along the coast off the State of São Paulo, Brazil“, "Benthic Marine Biodiversity on the State of São Paulo", and "REVIZEE/South Score/Benthos", ranging from the intertidal zone to deep sea environments, with the goal of enlarging the taxonomic knowledge of these families on the Southeastern and Southern coast of Brazil.

Identifications were based on morphological characters, specimens were observed under stereo- and optic microscopes and, in some cases, scanning electron microscope, with the help of specialized literature. Identification keys and detailed descriptions, with illustrative tables, were elaborated for each species.

In the present work, 8386 specimens were identified, belonging to 15 genera and 21 species, of which 14 genera and 18 species belonging to the family Terebellidae, subfamilies Terebellinae, Thelepodinae and Polycirrinae, and 1 genus and 3 species belonging to the family Trichobranchidae. Of those, 6 species of terebellids were new to science: Amaeana sp., Phisidia rubra Nogueira \& Alves, 2006, Polycirrus sp., Pseudostreblosoma brevitentaculatum Nogueira \& Alves, 2006, Terebella sp. 1 and Terebella sp. 2. The genus Phisidia Saint-Joseph, 1894 is new occurrence to Brazilian coast.

Thus, the present Dissertation can be used as an identification guide to help researchers, not only specialists, in the identification of part of the species of Terebellidae and Trichobranchidae occurring on the Southeastern-Southern regions of Brazil. It is also supposed to help researchers with the identification of species from localities outside the geographic range of the three projects here investigated, as well as further ecological and biogeographic studies, enlarging the knowledge of the biodiversity of Terebellidae and Trichobranchidae occurring in Brazil and worldwide. 


\section{INTRODUÇÃO}

A classe Polychaeta (filo Annelida) constitui um dos grupos de metazoários mais freqüentes e abundantes na fauna bentônica marinha, em todos os estratos batimétricos (Blake, 1997). Destacam-se pela sua diversidade e abundância nas associações bentônicas, nos mais diversos substratos, como costões rochosos, praias arenosas, fundos consolidados e não-consolidados, associando-se com esponjas, hidrozoários, moluscos, outros poliquetas, crustáceos, equinodermos, ascídias, algas e fanerógamas marinhas (Blankensteyn, 1988). São importantes em praticamente todos os ecossistemas bentônicos, tanto de regiões temperadas, quanto tropicais. Habitam, em sua maioria, as camadas superficiais do substrato, podendo participar ativamente na movimentação, reciclagem e aeração das camadas superficiais dos sedimentos (Amaral et al., 2004a). Por representarem aproximadamente um terço do número total de espécies do macrobentos, destacam-se no controle populacional de outros organismos e também são uma importante fonte alimentar para peixes, aves, crustáceos e outros organismos, participando significamente da rede trófica dos oceanos, principalmente das populações bentônicas (Amaral \& Migotto, 1980).

Existem aproximadamente 9000 espécies de Polychaeta formalmente descritas, distribuídas em cerca de 75 famílias (Rouse \& Pleijel, 2001). Os poliquetas são animais metamerizados (com corpo dividido em anéis), e cada segmento possui um par de parapódios, que são projeções laterais do corpo portadoras de cerdas. Originalmente, os parapódios são birremes, formados por notopódio, lobo dorsal, e neuropódio, lobo ventral, entretanto, muitas formas apresentam o padrão unirreme, com a redução dos notopódios.

Há animais errantes e sedentários, e, dentre os últimos, as famílias Terebellidae Grube, 1850 e Trichobranchidae Malmgren, 1866 são formadas por animais medindo de 5 a 300 mm de comprimento, tipicamente tubícolas, secretando tubos constituídos por uma camada de muco e partículas aglutinadas, como areia e fragmentos de pedras e conchas de moluscos. As principais exceções são os membros da subfamília Polycirrinae Malmgren, 1866, que não constroem tubos e vagueiam pelo substrato, e alguns membros do gênero Artacama Malmgren, 1866, que escavam o sedimento com o auxílio do peristômio, que forma um processo longo e cilíndrico (Kritzler, 1984a).

Os terebelídeos e tricobranquídeos são comedores de depósito seletivos (Fauchald \& Jumars, 1979), sendo facilmente reconhecidos no substrato através de seus numerosos tentáculos bucais elásticos, cobrindo uma ampla área ao redor da abertura do tubo, aglutinando, com muco, depósitos orgânicos, como bactérias e filmes de algas, que são conduzidos até a boca por sulcos ciliados nos tentáculos bucais (Hutchings, 2000a,b). Os lábios peristomiais são usados para selecionar as partículas de alimento antes da ingestão 
(Rouse \& Pleijel, 2001).

As duas famílias são registradas em todas as partes do mundo, desde as regiões costeiras até profundidades abissais, conforme registros taxonômicos e estudos biogeográficos (Holthe, 1986a,b; Garraffoni, 2006; Garraffoni et al., 2006). Apesar de algumas formas serem encontradas em locais profundos, como Unobranchus abyssalis Hartman, 1965, que foi registrado a 2700 metros de profundidade (Hartman, 1965a), a grande maioria vive em águas rasas (Imajima \& Williams, 1985), onde são facilmente encontradas superfícies sólidas, como rochas, fendas, conchas, esponjas e algas, locais em que costumam construir seus tubos (Kritzler, 1984a; Rouse \& Pleijel, 2001).

Os Terebellidae e Trichobranchidae são animais dióicos, com fecundação externa. O desenvolvimento pode ser direto ou indireto, com larva planctônica ou bentônica, no último caso, o desenvolvimento ocorre no interior do tubo parental ou em massas gelatinosas ao redor deste. Não há casos relatados de reprodução assexuada (Bhaud, 1988; Wilson, 1991; McHugh, 1993; Giangrande, 1997; Hutchings, 2000a,b; Garraffoni, 2006).

Terebella lapidaria Linnaeus, 1767 foi o primeiro terebelídeo a ser descrito. Posteriormente, Müller (1771) descreveu Amphitrite cirrata Müller, 1771, compondo o novo gênero Amphitrite Müller, 1771. Grube (1850) foi o primeiro autor a conferir o status de família aos terebelídeos, chamando-os de Terebellacea e incluindo os gêneros Terebella Linnaeus, 1767, Polycirrus Grube, 1850 e Terebellides Sars, 1835. A primeira grande revisão da família Terebellacea foi realizada por Malmgren (1866), que também descreveu novos gêneros e espécies, e dividiu as formas então conhecidas em cinco subfamílias: Amphitritea, Artacamacea (contendo o gênero Artacama), Canephoridea (contendo o gênero Terebellides), Polycirridea e Trichobranchidea (contendo o gênero Trichobranchus Malmgren, 1866). Posteriormente, Hessle (1917) adotou a terminologia Terebellidae para a família, e também alterou para -inae a terminação das subfamílias, reconhecendo Amphitritinae Malmgren, 1866, em que foi incluído o gênero Artacama, e Polycirrinae, e elevando Trichobranchinae ao nível de família (Trichobranchidae), incluindo Terebellides e Trichobranchus neste último grupo; neste sistema, Artacamacea e Canephoridea foram eliminadas. Além disso, Hessle (1917) também descreveu a subfamília Thelepinae de Terebellidae, cuja grafia foi posteriormente corrigida por Holthe (1976), passando para a forma correta Thelepodinae Hessle, 1917. Hessle (1917) ainda descreveu novos gêneros, sinonimizou outros e acrescentou diversos outros gêneros previamente descritos em Terebellidae, assim como incluiu o gênero Octobranchus Marion \& Bobretzsky, 1875 na família Trichobranchidae.

Fauvel (1927) dividiu novamente a família Trichobranchidae em duas subfamílias de Terebellidae, Trichobranchinae e Canephorinae, e revalidou a subfamília Artacaminae. Day (1967) sinonimizou as subfamílias Canephorinae com Trichobranchinae e Artacaminae com 
Amphitritinae. Portanto, com o último autor, Terebellidae passou a conter as subfamílias Amphitritinae (chamada por ele de Terebellinae, porém o nome não foi comumente usado por autores subseqüentes), Polycirrinae, Thelepodinae e Trichobranchinae. Hartmann-Schröder (1971) revalidou novamente a subfamília Artacaminae, retirando o gênero Artacama de Amphitritinae, e também reconheceu o status taxonômico de família para os Trichobranchidae. Posteriormente, autores como Rouse \& Pleijel (2001) e, mais recentemente, Garraffoni \& Lana (2004), Garraffoni et al., 2005 e Garraffoni (2006), propuseram a volta de Trichobranchidae para dentro de Terebellidae como uma subfamília, enquanto outros trabalhos mantêm aquele grupo com o status de família (Hartman, 1955; Holthe, 1986a,b; Solis-Weiss et al., 1991; McHugh, 1995; Fauchald \& Rouse, 1997; Rouse \& Fauchald, 1997; Hilbig, 2000a,b; Hutchings \& Peart, 2000; Colgan et al., 2001; Rousset et al., 2003, 2007; Glasby et al., 2004; Bartolomaeus et al., 2005; presente Dissertação). McHugh (1995), após uma análise cladística, incluiu o gênero Artacama em Amphitritinae, eliminando assim a subfamília Artacaminae, o que é consenso entre os autores na atualidade, e passou a chamar os Amphitritinae de Terebellinae, nomenclatura reconhecida como correta, e que passou a ser usada pelos autores nos trabalhos subseqüentes. Portanto, seguindo as propostas dos autores mais tradicionais, atualmente Terebellidae possui três subfamílias, Terebellinae, Thelepodinae e Polycirrinae, enquanto que Trichobranchidae é mantido com o status de família.

Terebellides stroemii Sars, 1835 foi o primeiro tricobranquídeo a ser descrito, sendo a família Trichobranchidae originalmente proposta por Hessle (1917), a fim de incluir os gêneros Trichobranchus, Terebellides e Octobranchus, como citado anteriormente. O autor também incluiu Ampharetides Ehlers, 1913 na família e sinonimizou Filibranchus Malm, 1874 com Trichobranchus. Fauchald (1977) revalidou Filibranchus e transportou Novobranchus Berkeley \& Berkeley, 1954, originalmente descrito como um gênero de Terebellidae, para Trichobranchidae, que passou a conter sete gêneros considerados válidos, Ampharetides, Filibranchus, Novobranchus, Octobranchus, Terebellides, Trichobranchus e Unobranchus Hartman, 1965. O gênero Artacamella Hartman, 1955, originalmente descrito como pertencente à subfamília Artacaminae, grupo atualmente incluído em Terebellinae, foi transferido para Trichobranchidae por Holthe (1977) e Hutchings (1977), independentemente. Posteriormente, Kingston \& Mackie (1980) colocaram Novobranchus em sinonímia com Octobranchus, e Holthe (1986a) também sinonimizou Ampharetides com Terebellides, e Filibranchus com Trichobranchus. Garraffoni \& Lana (2002) sustentaram a sinonímia de Filibranchus com Trichobranchus, e posteriormente os mesmos autores colocaram Artacamella em sinonímia com Trichobranchus (Garraffoni \& Lana, 2004). Dessa forma, Trichobranchidae passou a incluir os gêneros Octobranchus, Terebellides, Trichobranchus e Unobranchus (Garraffoni et al., 2005). 
Nas últimas décadas, houve um aumento significativo do número de gêneros e espécies novas das duas famílias, graças à publicação de diversos trabalhos envolvendo os grupos (Pearson, 1969; Hutchings, 1974, 1977, 1990, 1993, 1997a,b; Hutchings \& Rainer, 1979; Banse, 1980; Hutchings \& Murray, 1984; Kritzler, 1984a,b; Williams, 1984; Imajima \& Willians, 1985; Holthe, 1986a; Hutchings \& Glasby, 1986a,b,c, 1987, 1988, 1990, 1991; Blankensteyn \& Lana 1987; Tourtellotte \& Kritzler, 1988; Wang \& Wu, 1988; Hartmann-Schröder \& Rosenfeldt 1989; Zhirkov, 1989; Saphronova, 1991; Solis-Weiss et al., 1991; Hartmann-Schröder, 1992; Hsieh, 1994; Arvanitidis \& Koukouras, 1995; Rozbaczylo \& Mendez, 1996; Hutchings \& Smith, 1997; Bremec \& Elias, 1999; Hissmann, 2000; Hutchings \& Peart, 2000; Nogueira \& Amaral, 2001; Garraffoni \& Lana, 2003; Londoño-Mesa, 2003; Nogueira, 2003; Nogueira et al., 2003, 2004; Londoño-Mesa \& Carrera-Parra, 2005; Nogueira \& Alves, 2006; Nogueira \& Hutchings, 2007).

Atualmente, existem 483 espécies válidas de Terebellidae, distribuídas em 64 gêneros (Garraffoni, 2006; outros autores), e 58 espécies válidas de Trichobranchidae, distribuídas em 4 gêneros (Garraffoni et al., 2005).

As famílias Terebellidae e Trichobranchidae estão inseridas na ordem Terebellida, subordem Terebelliformia (Rouse \& Pleijel, 2001), também chamada Terebellomorpha (Holthe, 1986a), juntamente com as famílias Alvinellidae Desbruyères \& Laubier, 1979, Ampharetidae Malmgren, 1866 e Pectinariidae Quatrefages, 1865, segundo os resultados da análise cladística realizada por Rouse \& Fauchald (1997).

O posicionamento filogenético dos Terebellidae e Trichobranchidae dentro dos Terebellomorpha ainda é um assunto muito debatido, principalmente por não haver um consenso sobre a posição de Trichobranchidae, como família ou subfamília de Terebellidae. A dificuldade para reconhecer caracteres sinapomórficos que definam Terebellidae como grupo natural é observada nas análises filogenéticas do táxon, tanto de base morfológica quanto de base molecular, que utilizam um baixo número de representantes e um restrito conjunto de caracteres (Garraffoni, 2006).

McHugh (1995) indicou que a presença de almofadas glandulares ventrais consistia na apomorfia compartilhada pelas espécies da família Terebellidae. Porém, isso foi contestado, porque Ampharetidae e Pectinariidae também possuem almofadas glandulares ventrais (Fauvel, 1927; Rousset et al., 2003). De qualquer maneira, as almofadas não se encontram presentes nos membros de Trichobranchidae, que possuem região glandular ventral lisa, e, portanto, este caráter é usado na presente Dissertação para distinguir Terebellidae e Trichobranchidae.

Independentemente da análise e do tipo de caracteres utilizados, sejam morfológicos ou moleculares, apenas a subfamília Polycirrinae, dentre os Terebellidae, e os tricobranquídeos 
(não importando o seu status taxonômico) formam grupos monofiléticos (McHugh, 1995; Fauchald \& Rouse, 1997; Garraffoni \& Lana, 2004; Glasby et al., 2004; Garraffoni, 2006). A monofilia de Polycirrinae foi suportada pela presença de prostômio fundido ao lábio superior na forma de crista dorsal com expansões laterais, almofadas glandulares ventro-laterais pareadas e ausência de tubo. Já Trichobranchidae trata-se de um grupo monofilético com apomorfias que consistem em área glandular ventral lisa sem a distinção de almofadas e presença de uncini aciculares (Garraffoni, 2006).

A subfamília Thelepodinae sempre apareceu como não monofilética, uma vez que não foi encontrada nenhuma sinapomorfia capaz de sustentar o táxon, tanto em análises de base morfológica (Glasby et al., 2004; Garraffoni, 2006), quanto molecular (Colgan et al., 2001). Além disso, algumas espécies da subfamília compartilharam mais sinapomorfias, que no caso devem ser atribuídas a homoplasias, com espécies de Polycirrinae do que com as demais espécies de Thelepodinae (Garraffoni, 2006). Com relação a Terebellinae, a única característica considerada apomórfica na diagnose corrente da subfamília é a presença de fileiras duplas de uncini em, pelo menos, alguns segmentos torácicos. Porém, ainda há dúvidas quanto à monofilia do táxon, frente à grande quantidade de gêneros e sua diversidade morfológica. Garraffoni (2006) verificou que não existe suporte filogenético para a maioria dos gêneros de Terebellidae, uma vez que, segundo sua análise filogenética, as apomorfias que sustentam alguns gêneros estão ausentes nas diagnoses correntes dos mesmos. Isso indica que a formação da maioria dos gêneros está baseada em caracteres homoplásticos ou plesiomórficos, e, portanto, uma reclassificação dos gêneros com base em caracteres autapomórficos torna-se urgentemente necessária.

Existem estudos anatômicos morfológicos comparativos que utilizam estados de caracteres que podem ser usados para definir grupos (Orrhage, 2001; Zhadan \& Tzetlin, 2002, 2003), porém, a maioria dos estudos taxonômicos utiliza principalmente a morfologia externa para a identificação e descrição dos táxons.

Em Terebellidae e Trichobranchidae, a origem dos tentáculos bucais é prostomial, sendo o prostômio facilmente identificado como a região acima da banda do prototróquio na larva. $\mathrm{O}$ peristômio, região que contém o orifício bucal, é composto por duas partes distintas, o lábio superior e o lábio inferior. Segundo Zhadan \& Tzetlin (2002), os tentáculos bucais não possuem nenhuma conexão com a faringe ciliada, e, portanto, não são retráteis para dentro da boca, como ocorre em táxons próximos filogeneticamente dos terebelídeos e tricobranquídeos (Hilbig, 2000c). Segundo Garraffoni (2006), o correto entendimento da origem dos tentáculos bucais e das estruturas peristomiais é de suma importância para o esclarecimento das relações filogenéticas dentro e nos grupos próximos a Terebellidae e Trichobranchidae, e pode resultar na identificação de uma possível sinapomorfia. 
Nas duas famílias, o corpo é geralmente dividido em duas regiões distintas, tórax, com parapódios birremes (notopódios e neuropódios), e abdômen, apenas com neuropódios. Brânquias de morfologia e disposição variadas podem estar presentes na porção dorsal dos segmentos anteriores do tórax. Áreas glandulares estão presentes na superfície ventral do tórax, e correspondem à região produtora de muco, responsável pela aglutinação de partículas para a formação do tubo. No caso de Terebellidae, a superfície ventral glandular possui almofadas salientes ou é inflada lisa ou enrugada, enquanto que Trichobranchidae possui superfície glandular lisa. Convém ressaltar que inclusive as espécies da subfamília Polycirrinae, que não formam tubos e são organismos vágeis, possuem áreas glandulares desenvolvidas (Glasby \& Glasby, 2006).

Geralmente, o primeiro setígero do tórax possui apenas notopódios, e os neuropódios se originam a partir do segundo setígero ou mais posteriormente. A perda dos primeiros notopódios e neuropódios ao longo do desenvolvimento é um padrão recorrente em Terebellidae e Trichobranchidae, e acontece porque as cerdas típicas das fases iniciais do desenvolvimento desaparecem após um período, o que, segundo Garraffoni (2006), poderia ser um fato utilizado para delimitar o período larval. Na fase adulta, os notopódios portam notocerdas capilares de morfologia variada.

Os neuropódios possuem neurocerdas modificadas em forma de ganchos, com muitos dentes, chamadas de uncini, com função de ancorar o corpo do animal ao seu tubo, diminuindo assim os riscos de predação (Holthe, 1986b; Woodin \& Merz, 1987). Exceções estão presentes na família Terebellidae, dentre os gêneros Hauchiella Levinsen, 1893, Enoplobranchus Webster, 1879, Lysilla Malmgren, 1866, que não possuem neurocerdas, e Amaeana Hartman, 1959, em que as neurocerdas abdominais são em forma de espinhas, diferentes de uncini (esses gêneros estão inseridos na subfamília Polycirrinae), e também na família Trichobranchidae, no gênero Terebellides, em que o primeiro par de neuropódios torácicos apresenta espinhos aciculares, e os uncini aparecem a partir do segmento seguinte.

Os uncini podem estar dispostos em fileira única, curva em poucas espécies de Thelepodinae (formato de $\mathrm{C}$ ou em alça), ou dispostos em fileira dupla em alguns segmentos de Terebellinae, e neste caso as fileiras podem estar intercaladas ou com os uncini dispostos frente a frente ou costas com costas. Os gêneros Loimia Malmgren, 1866 e Lanice Malmgren, 1866 são considerados os únicos terebelíneos a possuírem fileiras duplas de uncini dispostos costas com costas, o que implicaria numa sinapomorfia compartilhada pelas espécies destes gêneros, mas o mesmo também ocorre em pelo menos uma espécie de Terebella (Capa \& Hutchings, 2006), e em Amphitrite lobocephala Hsieh, 1994 (Nogueira, comunicação pessoal).

Papilas nefridiais podem estar presentes nos segmentos 2-3, e papilas genitais podem estar presentes nos segmentos seguintes. Pelo menos no período reprodutivo, as papilas 
podem fornecer indícios de dimorfismo sexual. Benham (1927) observou em várias espécies que as papilas dos machos são mais visíveis e cilíndricas, enquanto que as das fêmeas são reduzidas a pequenas protuberâncias, enquanto que McHugh (1995) apenas encontrou esse padrão em alguns gêneros. Apesar desses estudos, a verificação do dimorfismo sexual nos espécimes é difícil, pois o formato das papilas pode variar de acordo com o grau de contração dos animais quando fixados, e geralmente as papilas são de difícil visualização na maioria dos exemplares, principalmente quando mal preservados.

No Brasil, já foram conduzidos diversos trabalhos contemplando as famílias (Nonato \& Luna, 1970; Rullier \& Amoureux, 1979; Lana, 1981; Nonato, 1981; Blankensteyn \& Lana, 1987; Blankensteyn, 1988; Morgado \& Amaral, 1989; Attolini, 1997, 2002; Sovierzoski, 1999; Garraffoni \& Lana, 2000, 2002; Nogueira, 2000, 2003; Nogueira \& Amaral, 2001; Garraffoni \& Costa, 2003; Nogueira et al., 2003, 2004; Garraffoni \& Camargo, 2006; Garraffoni et al., 2006; Nogueira \& Alves, 2006; dentre outros), tendo sido identificadas 34 espécies de Terebellidae até o momento, pertencentes a 20 gêneros (Garraffoni \& Costa, 2003; Nogueira, 2003; Nogueira et al., 2004; Amaral et al., 2006; Nogueira \& Alves, 2006), e 7 espécies de Trichobranchidae, pertencentes a 3 gêneros (Garraffoni \& Lana, 2003; Amaral et al., 2006). Entretanto, muitos destes registros constam de dissertações e teses não formalmente publicadas, com ênfase principalmente em ecologia e não contemplando descrições detalhadas dos táxons.

Aliado a isso, acredita-se atualmente que a ocorrência de espécies cosmopolitas de terebelídeos e tricobranquídeos, assim como ocorre em outras famílias de poliquetas, seja mal atribuída e que, na verdade, se tratem de complexos de espécies. Estudos conduzidos na Ásia e Oceania demonstram a ocorrência de espécies endêmicas naqueles locais (Hutchings, 1977, 1986, 1993, 1997a,b; Hutchings \& Rainier, 1979; Hutchings \& Murray, 1984; Hutchings \& Glasby, 1986a,b, 1987, 1988, 1990, 1991, 1995; Hutchings \& Smith, 1997; Hutchings \& Peart, 2000), e é provável que o mesmo ocorra no Brasil. Exemplos disso são discutidos na presente Dissertação, como no caso dos seguintes táxons de Terebellidae: Loimia medusa (Savigny, 1818), Loimia grubei (Grube, 1878) e Thelepus setosus (Quatrefages, 1865), que foram amplamente registradas no litoral brasileiro, mas provavelmente tratam-se de espécies novas para a ciência, uma vez que as localidades-tipo das mesmas são distantes do Brasil. Outro exemplo disso é verificado em Trichobranchidae, pois Terebellides stroemii, com localidadetipo na Noruega, tem sido amplamente reportada como cosmopolita. Williams (1984), Imajima \& Williams (1985), Solis-Weiss et al. (1991) e Hutchings \& Peart (2000) confirmaram que muitas espécies foram erroneamente reportadas como $T$. stroemii, e assim forneceram descrições e redescrições para diversos táxons.

De qualquer maneira, os padrões de distribuição de muitas espécies consideradas 
cosmopolitas são descontínuos, devido à escassez de registros ou levantamentos em diversas regiões do globo, inclusive a costa brasileira (Blankensteyn, 1988). Quando um grupo é focado em uma determinada região, muitas espécies novas são geralmente encontradas (Rouse \& Pleijel, 2001).

Atualmente, as faunas de poliquetas da África do Sul, Austrália, Mar do Norte, Europa e América do Norte são muito mais conhecidas do que nas demais regiões do globo (Hessle, 1917; Day, 1955, 1967; Hartman, 1955, 1965b; Hutchings, 1977, 1990, 1993, 1997a,b; Holthe, 1978, 1986a,b; Kritzler, 1984a,b; Hutchings \& Glasby, 1986a,b,c, 1987, 1988, 1991, 1995; Hilbig, 2000a,b,c), como extensas áreas da América do Sul e do continente africano que permanecem inexploradas quanto aos Terebellidae e Trichobranchidae (Garraffoni et al., 2006).

Até o momento, foram registradas cerca de 800 espécies de Polychaeta na costa brasileira, distribuídas em 60 famílias. Entre as diferentes regiões do Brasil, os estados de São Paulo, Paraná e Rio de Janeiro são os que possuem a fauna de poliquetas melhor conhecida, com mais de 460 espécies referidas para o Estado de São Paulo, que, tradicionalmente, mantém pesquisadores que se dedicam ao estudo do grupo, resultando num grande volume de trabalhos. Apesar do grau de conhecimento, persiste o problema do reduzido número de estudos taxonômicos e sinecológicos, sendo estes, em sua maioria, contribuições isoladas (Amaral et al., 2006), e dessa forma as regiões Sudeste e Sul ainda permanecem em um estágio de conhecimento que pode ser considerado incipiente (Lana et al., 1996).

$\mathrm{Na}$ costa brasileira, inclusive nas regiões Sudeste e Sul, ainda há diversos habitats que praticamente não foram explorados, como costões rochosos e ambientes de profundidade, sendo, de forma geral, poucos os estudos sobre a fauna bentônica realizados na região da plataforma externa (abaixo da isóbata de 50 metros), e quase inexistentes entre esta e o talude (Amaral et al., 2004b; Amaral, 2005), estratos batimétricos considerados como grandes vazios em termos de conhecimento faunístico (Migotto \& Tiago, 1999). Tem-se como concepção geral que a fauna bentônica, especialmente nesta região de transição entre quebra de plataforma e talude continental, é presumivelmente abundante, bastante peculiar, rica e de grande importância para a produção pesqueira (Amaral, 2005).

Buscando ampliar o atual estado de conhecimento sobre os poliquetas nesses ambientes, a presente Dissertação teve como propósito fazer um levantamento taxonômico das famílias Terebellidae (subfamílias Terebellinae, Thelepodinae e Polycirrinae) e Trichobranchidae, através de material coletado pelos projetos "A Biodiversidade de Poliquetas (Annelida: Polychaeta) em Costões Rochosos ao Longo do Estado de São Paulo", "Temático BIOTA/FAPESP/Biodiversidade Bêntica Marinha no Estado de São Paulo (=Temático BIOTA/FAPESP/Bentos Marinho)" e "REVIZEE/Score Sul/Bentos", abrangendo desde a zona 
entremarés a ambientes de profundidade, a fim de enriquecer o conhecimento taxonômico destas famílias no litoral Sudeste e Sul do Brasil. Os programas "Biodiversidade Bêntica Marinha do Estado de São Paulo" (BIOTA/FAPESP/Bentos Marinho) e "Avaliação do Potencial Sustentável dos Recursos Vivos da Zona Econômica Exclusiva" (REVIZEE) foram implantados a fim de conhecer a biodiversidade marinha brasileira, para que este conhecimento seja direcionado à conservação, e à utilização racional dos recursos potencialmente exploráveis no Brasil (Steiner, 2005). Assim, espera-se contribuir para a orientação de políticas de monitoramento e avaliação do ecossistema marinho, principalmente no caso dos ecossistemas de profundidade, que atualmente são muito utilizados para as atividades de prospecção e extração de petróleo (Amaral et al., 2004b).

Portanto, na presente Dissertação, estão disponíveis descrições detalhadas e ilustrações diagnósticas de todas as espécies encontradas. Dessa forma, este trabalho poderá ser utilizado por pesquisadores, inclusive não-especialistas, para identificar parte das espécies de Terebellidae e Trichobranchidae das regiões Sudeste-Sul do Brasil. Espera-se também que auxilie pesquisadores interessados em identificar espécies de locais não abrangidos pelos três projetos, assim como em futuros estudos ecológicos e biogeográficos, ampliando, assim, o conhecimento dos Terebellidae e Trichobranchidae presentes no Brasil e no mundo. 


\section{CONSIDERAÇÕES SOBRE A DISTRIBUIÇÃO E OCORRÊNCIA DAS ESPÉCIES ENCONTRADAS}

$\mathrm{Na}$ presente avaliação da distribuição das espécies encontradas, sua ocorrência será dada com relação a três ambientes:

1-) região entremarés: engloba costão rochoso e fital dos Projetos "Biodiversidade de Poliquetas" e "BIOTA".

2-) profundidades de 5 a 45,1 metros: engloba o infralitoral do Projeto "BIOTA".

3-) profundidades de 90 a 505 metros: engloba o Projeto "REVIZEE".

Dentre os Terebellinae, Phisidia rubra (22 espécimes) ocorre apenas na região entremarés, tendo sido encontrada apenas em Caraguatatuba (Praia Martim de Sá) e São Sebastião (Praias Araçá, Preta e Baleia). Amphitrite cf. variabilis conta com um único exemplar, proveniente de Ubatuba, coletado no infralitoral, a 21,5 m de profundidade. Terebella sp. 1 (61 espécimes) também ocorre apenas na região entremarés e foi encontrada em Ubatuba (Praia Picinguaba) e São Sebastião (Praia Araçá). Um exemplar de Terebella sp. 2 é proveniente do Estado de Rio de Janeiro, a 110 m de profundidade, e 29 exemplares são do Estado de São Paulo, encontrados nas profundidades de 147 m e 258 m. Em Caraguatatuba e São Sebastião, foram encontrados 2 espécimes de Artacama benedeni, a $44 \mathrm{~m}$ e 24,5 m, respectivamente, e no Estado de Rio de Janeiro foram encontrados 4 espécimes desta espécie, todos a $100 \mathrm{~m}$ de profundidade. Nicolea uspiana é extremamente abundante na região entremarés, estando presente em todos os locais amostrados, de Ubatuba a Peruíbe, inclusive ilhas, totalizando 7498 espécimes; apenas 6 espécimes foram encontrados no infralitoral, entre 6,1 m e 23,6 m, em Ubatuba. Loimia cf. grubei ocorre nos três ambientes aqui considerados, sendo que 4 espécimes são provenientes da região entremarés de Ubatuba (Praia Picinguaba) e Caraguatatuba (Praia Martim de Sá); 12 espécimes foram encontrados no infralitoral, entre 10,3 m e 44,5 m de profundidade, de Ubatuba a São Sebastião; e 2 espécimes são do Estado de Rio Grande do Sul, encontrados a 99 m e 100 m. Lanice sp. conta com um único exemplar encontrado no Estado de Rio de Janeiro, a $110 \mathrm{~m}$ de profundidade. Pista corrientis ocorre nos três ambientes aqui considerados, sendo que 15 exemplares são provenientes da região entremarés de Ubatuba (Praia Picinguaba), Caraguatatuba (Praia Martim de Sá), São Sebastião (Praias São Francisco e Baleia), e Santos (Ilha das Palmas); 19 exemplares foram encontrados no infralitoral, entre 8 m e 39,3 m, de Ubatuba a São Sebastião; e 30 exemplares são dos estados de Rio de Janeiro e São Paulo, encontrados entre 99 m e 157 m. Pista cf. cristata (41 espécimes) foi encontrada nos estados de Rio de Janeiro, São Paulo e Rio Grande do Sul, entre $90 \mathrm{~m}$ e $505 \mathrm{~m}$ de profundidade.

Dentre os Thelepodinae, Thelepus cf. setosus se distribui de Ubatuba a São Sebastião, 
no infralitoral, entre 16,2 m e 44,6 m de profundidade (45 exemplares); e 2 exemplares foram encontrados nos estados de Rio de Janeiro e São Paulo, a 110 m e 99 m, respectivamente. Pseudostreblosoma brevitentaculatum (35 espécimes) foi encontrada em Ubatuba e São Sebastião, no infralitoral, entre 9,3 m e $45 \mathrm{~m}$ de profundidade. É interessante notar que Streblosoma porchatensis (66 espécimes) foi encontrada apenas na região entremarés da Ilha Porchat (São Vicente) e da Ilha das Palmas (Santos). Streblosoma oligobranchiatum conta com 405 espécimes coletados na região entremarés de Ubatuba (Praia Picinguaba), Caraguatatuba (Praia Martim de Sá), e São Sebastião (Praias São Francisco, Baleia, Preta e Barequeçaba); e 9 espécimes são provenientes do infralitoral, de Ubatuba a São Sebastião, entre 25 m e 43,8 m de profundidade.

Com relação a Polycirrinae, apenas 1 exemplar de Lysilla sp. 1 foi encontrado em Ubatuba, a 21,5 m de profundidade; 2 exemplares de Lysilla sp. 2 foram coletados nos estados de Rio de Janeiro e São Paulo, a 254 m e 122 m de profundidade, respectivamente; e 1 exemplar de Amaeana sp. foi obtido em Ubatuba, a $35 \mathrm{~m}$ de profundidade. Polycirrus sp. foi encontrada na região entremarés (21 espécimes) de Ubatuba (Praia Picinguaba) e São Sebastião (Praias São Francisco e Araçá); e 3 espécimes foram encontrados no infralitoral, a 6,8 m, $9 \mathrm{~m}$ e 34,2 $\mathrm{m}$ de profundidade, em Ubatuba.

Finalmente, dentre os Trichobranchidae, Terebellides anguicomus conta com 11 exemplares obtidos de $5 \mathrm{~m}$ a 45,1 m de profundidade, em Ubatuba e São Sebastião. Apenas 2 espécimes de Terebellides sepultura foram encontrados, coletados a $147 \mathrm{~m}$ e $275 \mathrm{~m}$, no Estado de Rio de Janeiro. Terebellides lanai foi encontrada de Ubatuba a São Sebastião, entre 16,6 m e $45 \mathrm{~m}$ de profundidade (26 exemplares); e nos estados de Rio de Janeiro e Rio Grande do Sul, entre $93 \mathrm{~m}$ e $325 \mathrm{~m}$ de profundidade (9 exemplares).

Portanto, a região entremarés (cidades de Ubatuba a Peruíbe) apresentou a menor diversidade, com 8 espécies registradas, totalizando 8092 indivíduos (Phisidia rubra, Terebella sp. 1, Nicolea uspiana, Loimia cf. grubei, Pista corrientis, Streblosoma porchatensis, Streblosoma oligobranchiatum e Polycirrus sp.). A maior diversidade foi encontrada em profundidades de 5 a 45,1 metros (cidades de Ubatuba a São Sebastião), em que foram registradas 13 espécies, totalizando 171 indivíduos (Amphitrite cf. variabilis, Artacama benedeni, Nicolea uspiana, Loimia cf. grubei, Pista corrientis, Thelepus cf. setosus, Pseudostreblosoma brevitentaculatum, Streblosoma oligobranchiatum, Lysilla sp. 1, Amaeana sp., Polycirrus sp., Terebellides anguicomus, Terebellides lanai). E, em profundidades de 90 a 505 metros (estados de Rio de Janeiro, São Paulo e Rio Grande do Sul), foram registradas 10 espécies, totalizando 123 indivíduos (Terebella sp. 2, Artacama benedeni, Loimia cf. grubei, Lanice sp., Pista corrientis, Pista cf. cristata, Thelepus cf. setosus, Lysilla sp. 2, Terebellides sepultura, Terebellides lanai). 
Notavelmente, a região entremarés apresenta maior abundância, não somente pelo grande número de espécimes encontrados de Nicolea uspiana (7498) e Streblosoma oligobranchiatum (405), mas também em relação ao número de exemplares das demais espécies, que somam 189 indivíduos, quantidade ainda maior que o número encontrado nas duas faixas de profundidades. Vale destacar que a família Trichobranchidae não foi registrada, no presente trabalho, na região entremarés. 


\section{REFERÊNCIAS BIBLIOGRÁFICAS}

ABREU, J. 1978. Ecologia e distribuição dos Polychaeta e Mollusca na Enseada da

Fortaleza (Ubatuba, Estado de São Paulo). Tese de Doutorado. Instituto Oceanográfico, Universidade de São Paulo. 77 p.

AMARAL, A. C. Z. 1977. Anelídeos poliquetas do infralitoral em duas enseadas da região de

Ubatuba. Aspectos ecológicos. Tese de Doutorado. Instituto Oceanográfico, Universidade de São Paulo. 137 p.

AMARAL, A. C. Z. 1980. Anelídeos poliquetos do infralitoral em duas enseadas da região de Ubatuba. II. Aspectos ecológicos. Boletim do Instituto Oceanográfico, São Paulo, 29(1): 69-87.

AMARAL, A. C. Z. 2005. Introdução. In: Amaral, A. C. Z., Rizzo, A. E. \& Arruda, E. P. (eds.).

Manual de Identificação dos Invertebrados Marinhos da Região Sudeste-Sul do Brasil,

Vol. 1. São Paulo: Editora da Universidade de São Paulo. 21-22 pp.

AMARAL, A. C. Z., DENADAI, M. R., TURRA, A. \& RIZZO, A. E. 2003. Intertidal Macrofauna in Brazilian Subtropical Tide-Dominate Sandy Beaches. Journal of Coastal Research, 35: 446455.

AMARAL, A. C. Z., LANA, P. C., RIZZO, A. E., STEINER, T. M., PARDO, E. V., SANTOS, C. S. G., CARVALHO, A. C., WAGNER, M. F. R., GARRAFFONI, A. S., BRASIL, A. C. S., RIBEIRO, Z., NOGUEIRA, J. M. M., ABBUD, A., ROSSI, M. C. S. \& FUKUDA, M. V. 2004a. Filo Annelida, Classe Polychaeta. In: Amaral, A. C. Z. \& Rossi-Wongtschowski, C. L. D. B. (eds.). Biodiversidade Bentônica da Região Sudeste-Sul do Brasil - Plataforma Externa e Talude Superior. Série documentos Revizee - Score Sul. São Paulo: Instituto Oceanográfico, USP. 114-125 pp.

AMARAL, A. C. Z., LANA, P. C., FernandeS, F. C. \& COIMBRA, J. C. 2004b. Parte I Caracterização do Ambiente e da Macrofauna Bentônica. In: Amaral, A. C. Z. \& RossiWongtschowski, C. L. D. B. (eds.). Biodiversidade Bentônica da Região Sudeste-Sul do Brasil - Plataforma Externa e Talude Superior. Série documentos Revizee - Score Sul. São Paulo: Instituto Oceanográfico, USP. 11-46 pp.

AMARAL, A. C. Z. \& MIGOTTO, A. E. 1980. Importância dos anelídeos poliquetas na alimentação da macrofauna demersal e epibentônica da região de Ubatuba. Boletim do Instituto Oceanográfico, São Paulo, 29(2): 31-35.

AMARAL, A. C. Z., MORGADO, E. H. \& HENRIQUES, S. A. 1998. Polychaeta (Annelida) from the brazilian coast. Sixth Internacional Polychaete Conference, Program \& Abstracts, 6: 62.

Amaral, A. C. Z., MORgado, E. H., LOPES, P. P., BelÚCIO, L. F., LeITE, F. P. P. \& FERREIRA, C. P. 1990. Composition and distribution of the intertidal macrofauna of sandy beaches on São Paulo coast. Anais do II Simpósio de Ecossistemas da Costa Sul e Sudeste 
Brasileira - Estrutura, Função e Manejo. Publicação ACIESP, São Paulo, 3(71): 258-279.

AMARAL, A. C. Z., MORGADO, E. H., PARDO, E. V. \& REIS, M. O. 1995. Estrutura da comunidade de poliquetos da zona entremarés em praias da llha de São Sebastião (SP). Publicação especial Instituto Oceanográfico, São Paulo, (11): 229-237.

AMARAL, A. C. Z., NALLIN, S. A. H. \& STEINER, T. M. 2006. Catálogo das espécies de Annelida Polychaeta do Brasil. Disponível em www.ib.unicamp.br/projbiota/bentos_ marinho/prod_cien/texto_poli.pdf (consultado em 22/01/2008).

AMARAL, A. C. Z., NONATO, E. F. \& PETTI, M. A. V. 1994. Contribution of the polychaetous annelids to the diet of some brazilian fishes. Mémoires du Muséum National d'Histoire Naturelle, 162: 331-337.

ARVANITIDIS, C. \& KOUKOURAS, A. 1995. Amphitritides kuehmanni sp. nov. (Polychaeta: Terebellidae, Amphitritinae) from the Aegean Sea, with comments on the genus Amphitritides Augener. Ophelia, 40: 219-227.

ATTOLINI, F. S. 1997. Composição e distribuição dos anelídeos poliquetas na plataforma continental da região da Baía de Campos, RJ, Brasil. Dissertação de Mestrado. Instituto Oceanográfico, Universidade de São Paulo. 122 p.

ATTOLINI, F. S. 2002. Padrões de distribuição de poliquetas macrobentônicos na região de plataforma externa e talude superior entre Cabo Frio (RJ) e Cabo de Santa Marta (SC), costa sudeste do Brasil. Tese de Doutorado. Instituto Oceanográfico, Universidade de São Paulo. 115 p.

BANSE, K. 1980. Terebellidae (Polychaeta) from the Northeast Pacific Ocean. Canadian Journal of Fisheries and Aquatic Sciences, 37(1): 20-40.

BARTOlOMAEUS, T., PURSCHKE, G. \& HAUSEN, H. 2005. Polychaete phylogeny based on morphological data - a comparison of current attempts. Hydrobiologia, 535/536: 341-356.

BENHAM, W. B. 1927. External sexual differences in the terebellid worms. Proceedings of Zoology Society of London, 1: 141-148.

BHAUD, M. 1988. The two planctonic larval periods of Lanice conchilega (Pallas, 1766) Annelida Polychaeta, a peculiar example of the irreversibility of evolution. Ophelia, 29: 141-152.

BLAKE, J. A. 1997. Introduction to the Polychaeta. In: Blake, J.A., Hilbig, B. \& Scott, P. H. (eds.).

Taxonomic Atlas of the Bentic Fauna of the Santa Maria Basin and Western Santa Barbara Channel. Volume 4 - The Annelida Part 1. Oligochaeta and Polychaeta: Phyllodocida (Phyllodocidae to Paralacydoniiae) $2^{\text {nd }}$ ed. Santa Barbara, California: Santa Barbara Museum of Natural History. 37-108 pp.

BLANKENSTEYN, A. 1988. Terebellidae e Trichobranchidae (Annelida: Polychaeta) da Costa

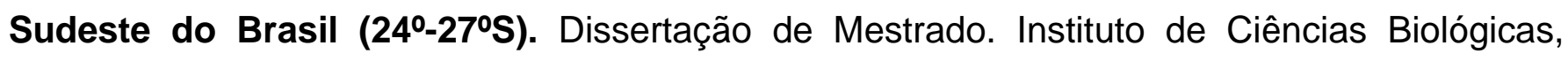
Universidade Federal do Paraná. 128 p. 
BLANKENSTEYN, A. \& LANA, P. C. 1987. Octobranchus longipes sp. n. (Trichobranchidae: Polychaeta) da costa sudeste do Brasil. Arquivos de Biologia e Tecnologia, 30: 671-676.

BLANKENSTEYN, A. \& MORENO, T. R. 1999. Nova ocorrência de Nicolea venustula (Montagu) (Polychaeta, Terebellidae) na costa sul do Brasil. Revista Brasileira de Zoologia, 16(1): 319322.

BREMEC, C. S. \& ELIAS, R. 1999. Species of Terebellides from South Atlantic waters off Argentina and Brazil (Polychaeta: Trichobranchidae). Ophelia, 51: 177-186.

CAPA, M. 2003. Estudio de la Criptofauna Coralina y de los Anélidos Poliquetos (Annelida: Polychaeta) de Substratos Duros del Parque Nacional de Coiba, Panamá. Tese de Doutorado. Universidad Autónoma de Madrid, Espanha. 382 p.

CAPA, M. \& HUTCHINGS, P. A. 2006. Terebellidae (Polychaeta) from Coiba National Park, Panamanian Pacific, including description of four new species and synonymy of the genus Paraeupolymnia with Lanicola. Zootaxa, 1375: 1-29.

COLGAN, D. J., HUTCHINGS, P. A. \& BROWN, S. 2001. Phylogenetic relationships within the Terebellomorpha. Journal of the Marine Biological Association of the United Kingdom, 81: 765773.

DAY, J. H. 1951. The Polychaeta Fauna of South Africa. Part I. The Intertidal and Estuarine Polychaeta of Natal and Mosambique. Annals of the Natal Museum, 11(1): 1-67.

DAY, J. H. 1955. The Polychaeta of South Africa. Part 3. Sedentary species from cape shores and estuaries. Journal of the Linnean Society of London, Zoology, 42: 407-452.

DAY, J. H. 1967. A Monograph on the Polychaeta of Soutern Africa. Part 2. Sedentaria. British Museum (Natural History). London. vii-xvii, 459-878 pp.

DAY, J. H. 1969. The Polychaet Fauna of South Africa. Part 6. Sedentary species dredged off cape coasts with a few new records from the shore. Journal of the Linnean Society of London, Zoology. 44(299): 463-560.

DAY, J. H. 1973. New Polychaeta from Beaufort, with a Key to All Species Recorded from North Carolina. NOAA Technical Reports NMFS CIRC-375. 140 p.

DUARTE, L. F. L. 1980. A endofauna da esponja Zygomicale parishii (Bowerbank) (Composição, dominância, diversidade e natureza da associação). Dissertação de Mestrado. Instituto de Biologia, Universidade Estadual de Campinas. 103 p.

DUARTE, L. F. L. \& NALESSO, R. C. 1996. The sponge Zygomicale parishii (Bowerbank) and its endobiotic fauna. Estuarine Coastal and Shelf Science, 42: 139-151.

FAUCHALD, K. 1977. The Polychaete Worms. Definitions and Keys to the Orders, Families and Genera. Natural History Museum of Los Angeles County. Science Series 28. 188 p.

FAUCHALD, K. \& JUMARS, P. A. 1979. The diet of worms: a study of polychaete feeding guilds. Oceanography and Marine Biology, an Annual Revue, 17: 193-284. 
FAUCHALD, K. \& ROUSE, G. W. 1997. Polychaete systematics: past and present. Zoologica Scripta, 26: 71-138.

FAUVEL, P. 1927. Polychètes Sédentaires. Addenda aux Errantes, Archiannélides, Myzostomaires. Faune de France, 16: 1-494.

FORNERIS, L. 1969. Fauna bentônica da Baía do Flamengo, Ubatuba. Aspectos ecológicos.

Tese de Livre-Docência. Instituto de Biociências, Universidade de São Paulo. 215 p.

FREIRE, O. 2004. Apresentação do Programa Revizee. In: Amaral, A. C. Z. \& RossiWongtschowski, C. L. D. B. (eds.). Biodiversidade Bentônica da Região Sudeste-Sul do Brasil - Plataforma Externa e Talude Superior. Série documentos Revizee - Score Sul. São Paulo: Instituto Oceanográfico, USP. 5-6 pp.

GARRAFFONI, A. R. S. 2006. Morfologia da região cefálica e dos uncini parapodiais, análise cladística e biogeografia da família Terebellidae (Annelida: Polychaeta). Tese de Doutorado. Instituto de Ciências Biológicas, Universidade Federal do Paraná. 153 p.

GARRAFFONI, A. R. S. \& CAMARGO, M. G. 2006. First Application of Morphometrics in a Study of Variations in Uncinial Shape Present within the Terebellidae (Polychaeta). Zoological Studies, 45(1): 75-80.

GARRAFFONI, A. R. S. \& COSTA, E. M. 2003. Two new species of Polycirrus (Polychaeta, Terebellidae) from Abrolhos Archipelago, Brazil. Zootaxa, 297: 1-7.

GARRAFFONI, A. R. S. \& LANA, P. C. 2000. Análise cladística do gênero Octobranchus Marion \& Bobretzky, 1875 (Trichobranchidae: Polychaeta). Notas técnicas da Facimar, 4: 43-48.

GARRAFFONI, A. R. S. \& LANA, P. C. 2002. Is Filibranchus Malm, 1874 (Trichobranchidae: Polychaeta) a natural taxon? Sarsia, 87: 472-477.

GARRAFFONI, A. R. S. \& LANA, P. C. 2003. Species of Terebellides (Polychaeta, Terebellidae, Trichobranchinae) from the Brazilian coast. Iheringia, 93(4): 355-363.

GARRAFFONI, A. R. S. \& LANA, P. C. 2004. Cladistic analysis of the subfamily Trichobranchinae (Polychaeta: Terebellidae). Journal of the Marine Biological Association of the United Kingdom, 84: 973-982.

GARRAFFONI, A. R. S., LANA, P. C. \& HUTCHINGS, P. A. 2005. A catalogue of Trichobranchinae (Polychaeta: Terebellidae) of the world. Zootaxa, 1065: 1-27.

GARRAFFONI, A. R. S., NIHEI, S. S. \& LANA, P. C. 2006. Distributions patterns of Terebellidae (Annelida: Polychaeta): an application of Parsimony Analysis of Endemicity (PAE). Scientia Marina, 70S3: 269-276.

GIANGRANDE, A. 1997. Polychaete reproduction patterns, life-cycles and life-histories: an overview. Oceanography and Marine Biology, 35: 323-386.

GLASBY, C. J. \& GLASBY, T. M. 2006. Two types of uncini in Polycirrus (Polychaeta: Terebellidae: Polycirrinae) revealed using geometric morphometrics. Journal of Natural History, . 
40(5-6): 237-253.

GLASBY, C. J., HUTCHINGS, P. A. \& HALL, K. 2004. Assessment of monophyly and taxon affinities within the polychaete clade Terebelliformia (Terebellida). Journal of the Marine Biological Association of the United Kingdom, 84: 961-971.

GRUBE, A. E. 1850. Die Familien der Anneliden. Archiv für Naturgeschichte, 16: 249-364.

HARTMAN, O. 1944. Polychaetous annelids from California, including the descriptions of two new genera and nine new species. Allan Hancock Pacific Expeditions, 10: 239-307.

HARTMAN, O. 1948. The marine annelids erected by Kinberg with notes on some other types in the Swedish State Museum. Arkiv for Zoologi, 42A(1): 1-37.

HARTMAN, O. 1955. Quantitative survey of the benthos of San Pedro Basin. Southern California. Part III. Systematic: Polychaeta. Allan Hancock Pacific Expeditions, 27: 1-86.

HARTMAN, O. 1965a. Deep-water benthic polychaetous annelids off New England to Bermuda and other North Atlantic areas. Allan Hancock Foundation Publications. Occasional Paper, 28: 1-378.

HARTMAN, O. 1965b. Catalogue of the polychaetous annelids of the world. Supplement 19601965 and index. Allan Hancock Foundation Publications, Occasional Paper, 23: 1-197.

HARTMAN, O. 1966a. Polychaeta Myzostomidae and Sedentaria of Antarctica. Antarctic Research Series, 7: IX+158 pp.

HARTMAN, O. 1966b. Polychaetous annelids of the Hawaiian Islands. Occasional Papers of Bernice P. Bishop Museum, 23(11): 163-252.

HARTMAN, O. 1969. Atlas of the sedentariate polychaetous annelids from California. Allan Hancock Foundation, Los Angeles. 812 p.

HARTMAN, O. 1978. Polychaeta from the Weddell Sea quadrant, Antarctica. Antarctic Research Series, 26: 125-223.

HARTMAN, O. \& FAUCHALD, K. 1971. Deep-water benthic polychaetous annelids off New England to Bermuda and other North Atlantic areas. Part II. Allan Hancock Monographs in Marine Biology, 6: 1-327.

HARTMANN-SCHRÖDER, G. 1962. Zur Kenntnis des Eulitorals der chilenischen Pazifikküste und der argentinischen Küste Südpatagoniens unter besonderer Berücksichtigung der Polychaeten und Ostracoden. Teil 2. Die Polychaeten des Eulitorals. Mitteilungen aus dem Hamburgischen zoologischen Museum und Institut, 60: 57-169.

HARTMANN-SCHRÖDER, G. 1965. Zur Kenntnis des Sublitorals der chilenischen Küste unter besonderer Berücksichtigung der Polychaeten uns Ostracoden. Teil 2. Die Polychaeten des Sublitorals. Mitteilungen aus dem Hamburgischen zoologischen Museum und Institut, 62: 59305.

HARTMANN-SCHRÖDER, G. 1971. Annelida, Borstenwürmer, Polychaeta. Die Tierwelt 
Deutschlands und der angrenzenden Meeresteile nach ihren Merkmalen und nach ihrer Lebensweise, 58: 1-594.

HARTMANN-SCHRÖDER, G. 1974a. Zur Kenntnis des Eulitorals der afrikanischen Westküste zwischen Angola und Kap der Guten Hoffnung und der afrikanischen Ostküste von Südafrika und Mocambique unter besonderer Berücksichtigung der Polychaeten und Ostracoden. Teil 2. Die Polychaeten des Untersuchungsgebietes. Mitteilungen aus dem Hamburgischen zoologischen Museum und Institut, 69: 95-228.

HARTMANN-SCHRÖDER, G. 1974b. Weitere Polychaeten von Ostafrika (Moçambique und Tansania). Mitteilungen aus dem Hamburgischen zoologischen Museum und Institut, 71: 23-33. HARTMANN-SCHRÖDER, G. 1979. Zur Kenntnis des Eulitorals der australischen Küsten unter besonderer Berücksichtigung der Polychaeten und Ostracoden. Teil 2. Die Polychaeten der tropischen Nordwestküste Australiens (zwischen Derby im Norden und Port Hedland im Süden). Mitteilungen aus dem Hamburgischen zoologischen Museum und Institut, 76: 77-218.

HARTMANN-SCHRÖDER, G. 1981. Zur Kenntnis des Eulitorals der australischen Küsten unter besonderer Berücksichtigung der Polychaeten und Ostracoden. Teil 6. Die Polychaeten der tropisch-subtropischen Westküste Australiens (zwischen Exmouth im Norden und Cervantes im Süden). Mitteilungen aus dem Hamburgischen zoologischen Museum und Institut, 78: 19-96.

HARTMANN-SCHRÖDER, G. 1986. Zur Kenntnis des Eulitorals der australischen Küsten unter besonderer Berücksichtigung der Polychaeten und Ostracoden. Teil 12. Die Polychaeten der antiborealen Südküste Australiens (zwischen Wallaroo im Westen und Port MacDonnell im Osten). Mitteilungen aus dem Hamburgischen zoologischen Museum und Institut, 83: 31-70.

HARTMANN-SCHRÖDER, G. 1992. Zur Polychaeten Fauna der Polynesischen Inseln Huahiné (Gesellschaftinseln) und Rangiroa (Tuamotu-Inseln). Mitteilungen aus dem Hamburgischen zoologischen Museum und Institut, 89: 49-84.

HARTMANN-SCHRÖDER, G. 1996. Annelida, Borstenwürmer, Polychaeta. 2, neubearbeitete Auflage. Tierwelt Deutschlands. 58: 648 p.

HARTMANN-SCHRÖDER, G. \& ROSENFELDT, P. 1989. Die Polychaeten der "Polarstern" -

Reise ANT III/2 in die Antarktis 1984. Teil 2. Cirratulidae bis Serpulidae. Mitteilungen aus dem Hamburgischen zoologischen Museum und Institut, 86: 65-106.

HESSLE, C. 1917. Zur Kenntnis der terebellomorphen Polychaeten. Zoologiska bidrag fran Uppsala, 5: 39-258.

HILBIG, B. 2000a. Family Terebellidae Grube, 1851. In: Blake, J.A., Hilbig, B. \& Scott, P. H. (eds.). Taxonomic Atlas of the Benthic Fauna of the Santa Maria Basin and Western Santa Barbara Channel. Volume 7 - The Annelida Part 4. Polychaeta: Flabelligeridae a Sternaspidae. Santa Barbara, California: Santa Barbara Museum of Natural History. 230-293 pp. 
HILBIG, B. 2000b. Family Trichobranchidae Malmgren, 1866. In: Blake, J.A., Hilbig, B. \& Scott, P. H. (eds.). Taxonomic Atlas of the Benthic Fauna of the Santa Maria Basin and Western Santa Barbara Channel. Volume 7 - The Annelida Part 4. Polychaeta: Flabelligeridae a Sternaspidae. Santa Barbara, California: Santa Barbara Museum of Natural History. 295-309 $\mathrm{pp}$.

HILBIG, B. 2000c. Family Ampharetidae Malmgren, 1866. In: Blake, J.A., Hilbig, B. \& Scott, P. H. (eds.). Taxonomic Atlas of the Benthic Fauna of the Santa Maria Basin and Western Santa Barbara Channel. Volume 7 - The Annelida Part 4. Polychaeta: Flabelligeridae a Sternaspidae. Santa Barbara, California: Santa Barbara Museum of Natural History. 169-230 pp.

HISSMANN, K. 2000. Lanice arakani, a new species of the family Terebellidae (Polychaeta:

Sedentaria) from seamounts of the West Mariana Ridge. Journal of Marine Association of United Kingdom, 80: 249-257.

HOLTHE, T. 1976. Paramphitrite tetrabranchia gen. et sp. nov. A new terebellid polychaete from western Norway, Sarsia. 61: 59-62.

HOLTHE, T. 1977. The systematic position of Artacamella Hartman, 1955 (Polychaeta, Terebellomorpha). Sarsia, 63: 35-37.

HOLTHE, T. 1978. The Zoogeography of the Terebellomorpha (Polychaeta) of the Northern European Waters. Sarsia, 63(3): 191-198.

HOLTHE, T. 1986a. Polychaeta Terebellomorpha. Marine Invertebrates of Scandinavia, 7: 1-194.

HOLTHE, T. 1986b. Evolution, systematics, and distribution of the Polychaeta Terebellomorpha, with a catalogue of the taxa and a bibliography. Gunneria, 55: 1-236.

HSIEH, H. L. 1994. Amphitrite lobocephala, a new species (Polychaeta: terebellidae) from Taiwan.

Proceedings of the Biological Society of Washington, 107: 517-532.

HUTCHINGS, P. A. 1974. Polychaeta of Wallis Lake, New South Wales. Proocedings of the Linnean Society of New South Wales, 98: 195-195.

HUTCHINGS, P. A. 1977. Terebelliform polychaeta of the families Ampharetidae, Terebellidae and Trichobranchidae from Australia, Chiefly from Moreton Bay, Queensland. Records of the Australian Museum, 31(1): 1-38.

HUTCHINGS, P. A. 1986. Terebellidae (Polychaeta) from the Hong Kong region. In: Morton, B.

(ed.). Marine Biological Workshop: The Marine Flora and Fauna of Hong Kong and Southern China. Hong Kong University Press. 377-412 pp.

HUTCHINGS, P. A. 1990. Terebellidae (Polychaeta) from the Hong Kong region. In: Morton, B.

(ed.). Proceedings of the Second International Marine Biological Workshop: The Marine Fauna and Flora of Hong Kong and Southern China. Hong Kong University Press: 377-411 pp. 
HUTCHINGS, P. A. 1993. New species of the family Terebellidae (Polychaeta) from Rottnest Island, Western Australia. In: Wells, F. E., Walker, D. I., Kirkman H. \& Lethbridge, R. (eds.). The marine flora and fauna of Rottnest Island, Western Australia. Western Australian Museum. 321-330 pp.

HUTCHINGS, P. A. 1997a. The Terebellidae (Polychaeta) of Northern Australia with a key to all the described species of the region. In: Hanley, J.R., Caswell, G., Megirian, D. \& Larson, H. K. (eds). The marine flora and fauna of Darwin Harbour, Northern Territory, Australia. Museums and Art Galleries of the Northern Territory and the Australian Marine Sciences Association. 133-161 pp.

HUTCHINGS, P. A. 1997b. The Terebellidae (F. Polychaeta) from the Wallabi Group, Houtman Abrolhos Islands, Western Australia. In: Wells, F.E. (ed.). The marine flora and fauna of the Houtman Abrolhos Islands, Western Australia. Western Australian Museum. 459-501 pp.

HUTCHINGS, P. A. 2000a. Family Terebellidae. In: Beesley, P. L., Ross, G. L. B. \& Glasby, C. J.

(eds.). Polychaeta \& Allies: The Southern Synthesis. Fauna of Australia. Vol. 4A. Polychaeta, Myzostomida, Pogonophora, Echiura, Sipuncula. Melbourne: CSIRO Publishing. 226-232 pp.

HUTCHINGS, P. A. 2000b. Family Trichobranchidae. In: Beesley, P. L., Ross, G. L. B. \& Glasby, C. J. (eds.). Polychaeta \& Allies: The Southern Synthesis. Fauna of Australia. Vol. 4A. Polychaeta, Myzostomida, Pogonophora, Echiura, Sipuncula. Melbourne: CSIRO Publishing. 232-235 pp.

HUTCHINGS, P. A. \& GLASBY, C. 1986a. A revision of the genus Euthelepus (Terebellidae:

Thelepinae). Records of the Australian Museum, 38(2): 105-117.

HUTCHINGS, P. A. \& GLASBY, C. 1986b. The Polycirrinae (Polychaeta: Terebellidae) from Australia. Records of the Australian Museum, 38(6): 319-350.

HUTCHINGS, P. A. \& GLASBY, C. 1986c. Glossothelepus, a new genus of Thelepinae (Polychaeta: Terebellidae) from the Gulf of California, Mexico. Proceedings of the Biological Society of Washington, 99(1): 84-87.

HUTCHINGS, P. A. \& GLASBY, C. 1987. The Thelepinae (Terebellidae) from Australia, with a discussion of the generic and specific characteres of the subfamily. Bulletin of the Biological Society of Washington, 7: 217-250

HUTCHINGS, P. A. \& GLASBY, C. 1988. The Amphitritinae (Polychaeta: Terebellidae) from Australia. Records of the Australian Museum, 40(1): 1-60.

HUTCHINGS, P. A. \& GLASBY, C. 1990. Additional new species of the family Terebellidae (Polychaeta) from Western Australia, with a key to all described species of the region. In: Wells, F. E., Walker, D. I., Kirkman, H. \& Lethbridge, R. (eds.). The marine flora and fauna of Albany, Western Australia. Western Australian Museum. 1: 251-289 pp. 
HUTCHINGS, P. A. \& GLASBY, C. 1991. Phylogenetic Implications of the Biogeography of Australian Terebellidae (Polychaeta). Ophelia, 5: 565-572.

HUTCHINGS, P. A. \& GLASBY, C. 1995. Description of the widely reported terebellid polychaetes Loimia medusa (Savigny) and Amphitrite rubra (Risso). Mitteilungen Aus Dem Hamburgischen Zoologischen Museum Und Institut, 92: 149-154.

HUTCHINGS, P. A. \& MURRAY, A. 1984. Taxonomy of polychaetes from the Hawkesbury River and the southern estuaries of New South Wales, Australia. Records of the Australian Museum, 36(3): 1-118.

HUTCHINGS, P. A. \& PEART, R. 2000. A revision of the Australian Trichobranchidae (Polychaeta). Invertebrate Taxonomy, 14: 225-272.

HUTCHINGS, P. A. \& RANIER, S. 1979. The polychaete fauna of the Careel Bay, Pittwater, New South Wales, Australia. Journal of Natural History, 13: 745-796.

HUTCHINGS, P. A. \& SMITH, R. I. 1997. Descriptions of new species and comments on previously described species of terebellid polychaetes from New Zealand and Australia. Bulletin of Marine Science, 60(2): 324-349.

IMAJIMA, M. \& HARTMAN, O. 1964. The polychaetous annelids of Japan. Part II. Allan Hancock Foudation Publications, 26: 239-452.

IMAJIMA, M. \& WILLIAMS, S. J. 1985. Trichobranchidae (Polychaeta) chiefly from the Sagami e Suruba Bays, collected by R/V Tansei Maru (Cruises KT - 65 - 76). Bulletin of the National Science Museum Series A, Zoology, 11(1): 7-18.

KINGSTON, P. F. \& MACKIE, A. S. Y. 1980. Octobranchus floriceps sp. nov. (Polychaeta: Trichobranchidae) from the northern North Sea with a re-examination of $O$. antarcticus Monro, 1936. Sarsia, 65: 249-254.

KRITZLER, H. 1984a. Chapter 52. Family Terebellidae Grube, 1850. In: Uebelacker, J. M. \& Johnson, P. G. (eds.). Polychaetes of the Northern Gulf of Mexico. Volume VII. Barry A. Vittor \& Associates, Inc., Mobile, Alabama. 52,1 - 52,72 pp.

KRITZLER, H. 1984b. Chapter 53. Family Trichobranchidae Malmgren, 1866. In: Uebelacker, J. M. \& Johnson, P. G. (eds.). Polychaetes of the Northern Gulf of Mexico. Volume VII. Barry A. Vittor \& Associates, Inc., Mobile, Alabama. 53,1 - 53,06 pp.

LANA, P. C. 1981. Padrões de Distribuição e Diversidade Específica de Anelídeos Poliquetos na Região de Ubatuba, Estado de São Paulo. Dissertação de Mestrado. Instituto Oceanográfico, Universidade de São Paulo. 111 p.

LANA, P. C. 1986. Macrofauna bêntica de fundos sublitorais não consolidados da Baía de Paranaguá (Paraná). Nerítica, Pontal do Sul, PR, 1(3): 79-89.

LANA, P. C., CAMARGO, M. G., BROGIM, R. A. \& ISAAC, V. J. 1996. O Bentos da Costa

Brasileira: Avaliação Crítica e Levantamento Bibliográfico. Rio de Janeiro, Femar. 432 p. 
LANGERHANS, P. 1880. Die Würmfauna von Madeira. Part 2. Zeitschrift für wissenschaftliche Zoologie, 33: 267-316.

LONDOÑO-MESA, M. H. 2003. Revision of Spinosphaera and establishment of the genus Hutchingsiella (Polychaeta: Terebellidae: Terebellinae). Journal of Marine Association of United Kingdom, 83: 747-759.

LONDOÑO-MESA, M. H. 2006. Revision of Paraeupolymnia, and redescription of Nicolea uspiana comb. nov. (Terebellidae: Polychaeta). Zootaxa, 1117: 21-35.

LONDOÑO-MESA, M. H. \& CARRERA-PARRA, L. F. 2005. Terebellidae (Polychaeta) from the Mexican Caribbean with description of four new species. Zootaxa, 1057: 1-44.

LOPES, P. P. 1993. Estrutura da comunidade de poliquetos da zona entremarés da região do Araçá, São Sebastião (SP). Dissertação de Mestrado. Instituto de Biologia, Universidade Estadual de Campinas. $106 \mathrm{p}$.

LUEDERWALDT, H. 1929. Resultados de uma excursão científica à ilha de São Sebastião no litoral do Estado de São Paulo e em 1925. Revista do Museu Paulista, 16: 1-79.

MACIEL, P. M. 1996. Distribuição e abundância dos anelídeos poliquetos e seu papel trófico

no ecossistema costeiro do Canal de São Sebastião, São Paulo (Brasil). Tese de Doutorado. Instituto Oceanográfico, Universidade de São Paulo. 130 p.

MALMGREN, A. J. 1866. Nordiska Hafs-Annulater. Öfversigt af Kongiliga Veteskaps-Akademiens Förhandlingar, 22: 355-410.

McHUGH, D. 1993. A comparative study of reproduction and development in the polychaete family Terebellidae. Biological Bulletin, 185: 153-168.

McHUGH, D. 1995. Phylogenetic analysis of the Amphitritinae (Polychaeta: Terebellidae). Zoological Journal of the Linnean Society, 114: 405-429.

MigotTO, A. E. \& TIAGO, C. G. 1999. Síntese. In: Migotto, A. E. \& Tiago, C. G. (eds). Biodiversidade do Estado de São Paulo, Brasil: Síntese do Conhecimento ao Final do Século XX. São Paulo: Fapesp, vol. 3, Invertebrados Marinhos. 301-310 pp.

MOHAMMED, M. B. M. 1973. New species and records of polychaete annelids from Kuwait, Arabian Gulf. Zoological Journal of the Linnean Society, 52: 23-44.

MORGADO, E. H. 1980. A Endofauna de Schizoporella unicornis (Johnston, 1847) (Bryozoa), no Litoral Norte do Estado de São Paulo. Dissertação de Mestrado. Instituto de Biologia, Universidade Estadual de Campinas. 126 p.

MORGADO, E. H. 1988. Anelídeos poliquetas do sublitoral da região de Ubatuba - SP, compreendida entre as Ilhas Anchieta e Vitória. Tese de Doutorado. Instituto de Biologia, Universidade Estadual de Campinas. $193 \mathrm{p}$.

MORGADO, E. H. \& AMARAL, A. C. Z. 1989. Anelídeos poliquetas da região de Ubatuba (SP): padrões de distribuição geográfica. Revista Brasileira de Zoologia, 3: 535-568. 
MORGADO, E. H., AMARAL, A. C. Z., BELÚCIO, L. F., LOPES, P. P., FERREIRA, C. P. \& LEITE,

F. P. P. 1990. The intertidal macrofauna of São Sebastião complex beaches (São Sebastião-

SP). Anais do II Simpósio de Ecossistemas da Costa Sul e Sudeste Brasileira - Estrutura, Função e Manejo. Publicação ACIESP, São Paulo, 3(71): 314-325.

MORGADO, E. H., AMARAL, A. C. Z., NONATO, E. F. \& SALVADOR, L. B. 1994. Intertidal sandy

beaches polychaetes of São Sebastião Island, southern Brazil. In: Dauvin, J. C., Laubier, L. \&

Reish, D. J. (eds.). Actes de la 4ème Conferénce Internationale des Polychètes. Mémoires

du Muséum National d`Histoire Naturelle. 162: 603-610 pp.

MÜLLER, F. 1858. Einiges uber die Anneliden-Fauna der Isel St. Catharina an der Brazilianischen

Kuste. Archiv fur Naturgeschichte, 24: 211-220.

MÜLLER, O. F. 1771. Von Würmern des süssen und salzigen Wassers. Copenhagen. 200 p.

MUNIZ, P., PIRES-VANIN, A. M. S., BURONE, L. \& SILVA, J. P. 1996. Density and distribution of

polychaetes in the infralitoral of Mar Virado Bight (Ubatuba, SP), southern brazilian coast. Anais

da Academia Brasileira de Ciências, 68(3): 453-463.

NOGUEIRA, J. M. M. 2000. Anelídeos Poliquetas Associados ao Coral Mussismilia hispida

(Verrill, 1868) em Ilhas do Litoral do Estado de São Paulo. Phyllodocida, Amphinomida,

Eunicida, Spionida, Terebellida e Sabellida. Tese de Doutorado. Instituto de Biociências, Universidade de São Paulo. 265 p.

NOGUEIRA, J. M. M. 2003. A new species of Paraeupolymnia Young and Kritzler, 1986

(Polychaeta: Terebellidae: Terebellinae) from Brazil. Scientia Marina, 67(4): 403-411.

NOGUEIRA, J. M. M. \& ALVES, T. M. 2006. Two new terebellid polychaetes (Polychaeta:

Terebellidae) from the state of São Paulo, southeastern Brazil. Zootaxa, 1205: 31-54.

NOGUEIRA, J. M. M. \& AMARAL, A. C. Z. 2001. New terebellids (Polychaeta: Terebellidae) living

in colonies of a stony coral in the state of São Paulo, Brazil. Proceedings of the Biological Society of Washington, 114(1): 285-296.

NOGUEIRA, J. M. M., GARRAFFONI, A. R. S. \& ALVES, T. M. 2004. A new species of

Streblosoma Sars, 1872 (Polychaeta, Terebellidae, Thelepodinae) from Brazil, with comments

on Streblosoma oligobranchiatum Nogueira \& Amaral, 2001. Beaufortia, 54(7): 93-103.

NOGUEIRA, J. M. M. \& HUTCHINGS, P. A. 2007. New species of terebellid polychaetes

(Polychaeta: Terebellidae) from Australia. Zootaxa, 1473: 1-24.

NOGUEIRA, J. M. M., HUTCHINGS, P. A. \& AMARAL, A. C. Z. 2003. Articulatia, a new genus of

Terebellinae (Polychaeta, Terebellidae) living in brazilian corals. Journal of the Marine

Biological Association of the United Kingdom, 83: 761-770.

NONATO, E. F. 1973. Anelídeos poliquetas. Relatório sobre a segunda pesquisa oceanográfica e pesqueira do Atlântico Sul entre Torres e Maldonado (lat. $29^{\circ} \mathrm{S}-35^{\circ} \mathrm{S}$ ). Programa Rio Grande do Sul II, Parte I. Instituto Oceanográfico, Universidade de São Paulo. 
231-232 pp.

NONATO, E. F. 1981. Contribuição ao conhecimento dos anelídeos poliquetas bentônicos da plataforma continental brasileira, entre Cabo Frio e o Arroio Chuí. Tese de LivreDocência. Instituto Oceanográfico, Universidade de São Paulo. 246 p.

NONATO, E. F. \& LUNA, J. A. C. 1970. Anelídeos poliquetas do nordeste do Brasil. I. Poliquetas bentônicos da costa de Alagoas e Sergipe. Boletim do Instituto Oceanográfico, São Paulo, 19: 57-130.

ORENSANZ, J. M. \& GIANUCA, N. M. 1974. Contribuição ao conhecimento dos anelídeos poliquetas do Rio Grande do Sul. I. Lista sistemática preliminar e descrição de três espécies. Comunicações do Museu de Ciências PUCRGS, Porto Alegre, 4: 1-37.

ORRHAGE, L. 2001. On the anatomy of the central nervous system and the morphological value of the anterior end appendages of Ampharetidae, Pectinariidae and Terebellidae (Polychaeta). Acta Zoológica, 82: 57-71.

PAIVA, P. C. 1990. Padrões de distribuição e estrutura trófica dos anelídeos poliquetas da Plataforma Continental do litoral norte do Estado de São Paulo. Dissertação de Mestrado. Instituto Oceanográfico, Universidade de São Paulo. 146 p.

PAIVA, P. C. 1993. Trophic structure of a shelf polychaete taxocoenosis in southern Brazil. Cahiers de Biologie Marine, 35: 39-55.

PAIVA, P. C. 1996. Variação espacial e temporal da macrofauna bentônica da Enseada de Picinguaba, Ubatuba, SP: relevância no planejamento amostral em estudos oceanográficos e de monitoramento ambiental de fundos marinhos consolidados. Tese de Doutorado. Instituto Oceanográfico, Universidade de São Paulo. 140 p.

PARDO, E. V. 1995. Padrões de distribuição e estrutura trófica dos poliquetos da região entremarés de praias da Ilha de São Sebastião (Ilhabela, SP). Dissertação de Mestrado. Instituto de Biociências, Universidade Estadual Paulista. 77 p.

PEARSON, T. H. 1969. Scionella lornensis sp. nov., a new terebellid (Polychaeta: Annelida) from the west coast of Scotland, with notes on the genus Scionella Moore, and a key to the genera of the Terebellidae recorded from European waters. Journal Natural History, 3: 509-516.

PETTI, M. A. V. \& NONATO, E. F. 2000. Temporal variation of Polychaetes from Picinguaba and Ubatumirim bights (southeastern Brazil). Bulletin of Marine Science, 67(1): 127-136.

PIRES-VANIN, A. M. S., CORBISIER, T. N., ARASAKI, E. \& MÖELLMANN, A. M. 1997. Composição e distribuição espaço-temporal da fauna bêntica no Canal de São Sebastião. Relatórios Técnicos do Instituto Oceanográfico, 41: 29-46.

REIS, M. O. 1995. Estrutura e dinâmica da macrofauna bêntica de poliquetos da região entremarés de praias da Ilha de São Sebastião (SP). Dissertação de Mestrado. Instituto de Biologia, Universidade Estadual de Campinas. 81 p. 
REIS, M. O., MORGADO, E. H., DENADAI, M. R. \& AMARAL, A. C. Z. 2000. Polychaete zonation on sandy beaches of São Sebastião Island, São Paulo State, Brazil. Revista Brasileira de Oceanografia, 48(2): 107-117.

RIOJA, E. 1941. Estudios Anelidologicos. III. Datos para el conocimiento de la fauna de poliquetos de las costas del Pacifico de México. Anales del Instituto de Biología de México, 12(2): 669746.

RIOJA, E. 1947. Estudios Anelidologicos. XVII. Contribución al conocimiento de los anélidos poliquetos de Baja California y Mar de Cortes. Anales del Instituto de Biología de México, 18(1): 197-224.

RIOJA, E. 1959. Estudios Anelidologicos. XXII. Datos para el conocimiento de la fauna de anélidos poliquetos de las costas orientales de México. Anales del Instituto de Biología de México, 29(1-2): 219-301.

RIZZO, A. E. 1998. Composição e distribuição de anelídeos na região entremarés das praias São Francisco e Engenho D’água, Canal de São Sebastião (SP). Dissertação de Mestrado. Instituto de Biociências, Universidade Estadual Paulista. 74p.

RIZZO, A. E. \& AMARAL, A. C. Z. 2000. Temporal variation of annelids in the intertidal zone of beaches of the São Sebastião Channel, southern Brazil. Journal of the Marine Biological Association of the United Kingdom, 80: 1007-1017.

RIZZO, A. E. \& AMARAL, A. C. Z. 2001. Environmental variables and intertidal beach annelids of São Sebastião Channel (State of São Paulo, Brazil). Revista de Biología Tropical, 49(3-4): 849857.

ROSSET, V., PleiJel, F., ROUSE, G. W., ERSÉUS, C. \& SIDDALL, M. E. 2007. A molecular phylogeny of annelids. Cladistics, 23: 41-63.

ROSSET, V., ROUSE, G. W., FERAL, J. P., DESBRUYÈRES, D. \& PLEIJEL, F. 2003. Molecular and morphological evidence of Alvinellidae relationships (Terebelliformia, Polychaeta, Annelida). Zoologica Scripta, 32: 185-197.

ROUSE, G. W. \& FAUCHALD, K. 1997. Cladistics and polychaetes. Zoologica Scripta, 26: 139204.

ROUSE , G. W. \& PLEIJEL, F. 2001. Polychaetes. Oxford University Press. 354 p.

ROZBACZYLO, N. \& MÉNDEZ, M. A. 1996. Artacama valparaisiensis, a new species of Terebellidae (Annelida: Polychaeta) from subtidal soft bottoms of Valparaiso Bay, Chile. Proceedings of the Biological Society of Washington, 109: 138-142.

RULLIER, F. \& AMOUREUX, L. 1979. Annelids Polychètes: campagne de la Calipso au large des côtes atlantiques de l' Amerique du Sud. Annales de l'Institute Oceanografique, 55: 145-206.

SANTA-ISABEL, L. M., LEÃO, Z. M. A. N. \& PESO-AGUIAR, M. C. 2000. Polychaetes Guarajuba coral reefs, Bahia, Brazil. Bulletin of Marine Science, 67(1): 645-653. 
SANTOS, M. A., SANTOS, C. S. G. \& OLIVEIRA, C. M. M. 1994. Polychaeta in the estuary of the Piauí River, Sergipe, Brazil. Mémoires du Muséum national d'histoire naturelle, 162: 541-547.

SAPHRONOVA, M. A. 1991. Redescription of some species of Scionella Moore, 1903, with a review of the genus and comments on some species of Pista Malmgren, 1866 (Polychaeta: Terebellidae). Ophelia, 5: 239-247.

SOLIS-WEISS, V., FAUCHALD, K. \& BLANKENSTEYN, A. 1991. Trichobranchidae (Polychaeta) from shallow warm water areas in the western Atlantic Ocean. Proceedings of the Biological Society of Washington, 104(1): 147-158.

SOUTHWARD, E. C. 1956. On some Polychaeta of the Isle of Man. Annals of the Magazine of Natural History, IX(12): 257-279.

SOVIERZOSKI, H. H. 1991. Estrutura temporal da comunidade macrobentônica da foz do

Rio Maciel, Baía de Paranaguá, Paraná. Dissertação de Mestrado. Instituto de Ciências Biológicas, Universidade Federal do Paraná. 98 p.

SOVIERZOSKI, H. H. 1999. Anelídeos Poliquetas do Litoral de Alagoas, Brasil. Tese de Doutorado. Instituto de Biociências, Universidade de São Paulo. 208 p.

STEINER, T. M. 2005. Estudo Taxonômico da Família Onuphidae (Annelida, Polychaeta) das

Regiões Sudeste e Sul do Brasil. Tese de Doutorado. Instituto de Biociências, Universidade de São Paulo. 210 p.

TOMMASI, L. R. 1967. Observações preliminares sobre a fauna bêntica de sedimentos moles da Baía de Santos e regiões vizinhas. Boletim do Instituto Oceanográfico, São Paulo, 16(1): 43-64. TOMMASI, L. R. 1979. Considerações ecológicas sobre o sistema estuarino de Santos (SP).

Tese de Livre-Docência. Instituto Oceanográfico, Universidade de São Paulo. Volumes I-II. 489 p.

TOURTELLOTTE, G. \& KRITZLER, H. 1988. Scionella papillosa, a new species of Polycheta (Polychaeta: Terebellidae) from the southwest Florida Continental Shelf. Proceedings of the Biological Society of Washington, 101: 79-82.

WANG, Y. H. \& WU, B. L. 1988. Two new species of Polychaeta from the first Chinese Antarctic and South Oceanic Expedition (Maldanidae, Trichobranchidae). Acta Zootaxonomica Sinica, 13: 229-233.

WILLIAMS, S. J. 1984. The status of Terebellides stroemii (Polychaeta: Trichobranchidae) as a cosmopolitan species, based on a worldwide morphological survey, including description of new species. In: Hutchings, P. A. (ed.). Proceedings of the First International Polychaete Conference, Sydney, Australia, 1984. The Linnean Society of New South Wales, Sydney. 118-142 pp.

WILSON, D. P. 1928. The post-larval development of Loimia medusa Sav. Journal of the Marine Biological Association of the United Kingdom, 15: 129-149. 
WILSON, W. H. 1991. Sexual reproductive modes in polychaetes: classification and diversity. Bulletin of Marine Science, 48: 500-516.

WOODIN, S. A. \& MERZ, R. A. 1987. Holding on by their hooks: anchors for worms. Evolution, 41(2): 427-432.

YOUNG, M. W. \& KRITZLER, H. 1987. Paraeupolymnia, a new genus of terebellid (Polychaeta:

Terebellidae) from Belize. Proceedings of Biological Society of Washington, 100(4): 687-690.

ZHADAN, A. E. \& TZETLIN, A. B. 2002. Comparative morphology of the feeding apparatus in the Terebellida (Annelida: Polychaeta). Cahiers de Biologie Marine, 43: 149-164.

ZHADAN, A. E. \& TZETLIN, A. B. 2003. Comparative study of the diaphragm (gular membrane) in Terebelliformia (Polychaeta, Annelida). Hydrobiologia, 496: 269-278.

ZHIRKOV, I. A. 1989. Donnaja fauna morei SSSR, polihet. Moscow: Moscow University. 141 p. 